\title{
A Modified Nested-Gridding for Upscaling-Downscaling in Reservoir Simulation
}

\author{
Masoud Babaei · Peter R. King
}

Received: 26 April 2011 / Accepted: 6 March 2012 / Published online: 5 April 2012

(C) Springer Science+Business Media B.V. 2012

\begin{abstract}
The upscaled models of reservoirs result in coarse representation of flow for simulation gridblocks. This representation discards subgrid heterogeneities and flow complexities which can lead to an inaccurate performance prediction for reservoir processes. To tackle the problem, reconstruction techniques such as multiscale or upscaling-downscaling methods have been developed to recapture the subgrid flow details which have been approximated at the upscaling stage. In this study, we propose a modified downscaling procedure applied on local-global upscaling method. The modification accounts for using approximate fine-scale velocities obtained from the upscaling stage to partition the coarse-scale fluxes to local boundary conditions in the reconstruction of fine-scale solutions. This modification enhances quality of saturation profiles obtained by resultant reconstructed velocities. In this stage, we also investigate the application of basis functions for velocity reconstruction. These functions are computed numerically by solving at initial time a set of decomposed Neumann boundary condition problems for each coarse block. The values of boundary fine fluxes are derived from apportioning a unit-value coarse flux over that boundary. These functions are stored and reused for the rest of the simulation. This leads to computational gain compared to conventional downscaling where velocity is refined at any time and any where. We simulate incompressible tracer and multiphase flow in two and three dimensions on a range of test cases by fine-scale reference model and upscaling-downscaling model. For comparison purposes a pressure solver upscaling is used as a base method. The results show a better reconstruction quality for saturation profiles obtained by modified scheme and the practicality of application of basis functions with minimal deterioration of results.
\end{abstract}

Keywords Upscaling · Downscaling · Flow reconstruction · Nested gridding · Multiphase flow

M. Babaei $(\varangle) \cdot$ P. R. King

Department of Earth Science \& Engineering, Imperial College London, Prince Consort Road, London SW7 2AZ, UK

e-mail: masoud.babaei08@imperial.ac.uk 


\section{Introduction}

The increased level of details in geological reservoir models (geomodels) has made the numerical simulation of flow of hydrocarbon for such models computationally expensive and time consuming. Nonetheless it is very interesting to enhance the precision and accuracy of prediction of flow by the use of detailed geomodels. Moreover developing optimal reservoir production strategies, considering the high uncertainty in reservoir properties, often requires many simulation runs. Therefore, a computationally efficient upscaling is still on high demand and that upscaling should be equally accurate enough for decision-making and process design purposes.

In upscaling, the smaller-size representations of reservoir models that best mimic the large size model in simulation output is required. In replacing a finely defined heterogeneous model with a coarser model, we calculate and assign homogenized properties to the coarse gridblocks based on the underlying fine grid values. Permeability is often the most important property to be upscaled, many permeability upscaling methods have been developed that concern either only absolute permeability for single phase (see Wen and Gómez-Hernández 1996; Renard and de Marsily 1997; Farmer 2002) or include relative permeability and capillary pressure as well for multiphase flow. For a review on relative permeability upscaling we refer to Barker and Thibeau (1997) and Christie (2001).

Upscaling often has the unintended side effects of introducing averaging error in sampling (or homogenizing) and truncation error due to the loss of resolution in coarse discretization. The sampling or homogenization error arises from representation of a group of fine values with an inappropriate coarse value. This error is more pronounced in the presence of highly correlated heterogeneities like channels or barriers. This error occurs because the scale at which the averaging is performed may not completely capture the actual fine flow behavior. Truncation error or numerical dissipation on the other hand is due to discretization error in representing continuum differentials. Dissipation smears sharp front movement of flow within a coarse block.

To decrease the errors, new techniques of upscaling and multiscale methods have been developed in recent decades. One significant improvement, for example, lies in broadening the sampling to include more information about the fine flow. For more description, we first discuss the classification of sampling methods. In the derivation of a single value for coarse permeability, we can either use analytical or numerical approaches. Analytical methods are suitable for only a few special patterns of heterogeneity and tend to become inaccurate outside the domain of their validity. So most often the numerical schemes are used to calculate an average value by imposing an assumed boundary condition over the area of sampling. Based on the domain on which the sampling is numerically performed and the boundary conditions which are assumed, numerical methods are classified as local, extended local, global, and local-global (or quasi global) methods.

The sampling in local methods is performed in a limited area within which the coarse permeability will be used. This localization may produce unsatisfactory results if large-scale connected paths exist within a reservoir. For improving the local numerical methods, we use jackets or oversampling to include a larger surrounding area (Gómez-Hernández and Journel 1990; Hou and Wu 1997). This will lead to an extended local calculation of upscaling values (Durlofsky 2005). Moreover, one can use the entire global information of the domain including the boundary conditions derived from global properties to enhance sampling. Examples include White and Horne (1987) that derives coarse-scale properties based on several global flows, or Holden and Nielsen (2000) that formulate the global upscaling based on an optimization problem for a given flow scenario. The disadvantage is then the cost of application of the 
global domain. Alternatively, one can use limited global information in a coupled framework of a local-global method. This is developed by Chen et al. (2003). First, a local or extended local upscaling is performed and an initial estimate of upscaled quantities is obtained. Then a global coarse pressure field is computed. These pressures are used to set boundary conditions on the new extended local problems and the process is repeated until consistency is reached. To guarantee computational efficiency, the iteration can be performed only in areas of high flow rates. This is suggested in Chen and Durlofsky (2006). This technique is a core part of this study so we will discuss it later in more details.

As mentioned earlier a group of numerical approaches to reduce the errors of upscaling is multiscale methods. Multiscale techniques refer to numerical schemes which model physical phenomena at a coarse scale while preserve underlying fine-scale features inside the coarse solution using partial differential equations and corresponding operators at different scale. Many multiscale algorithms have been proposed for flow in porous media in literature, the multiscale finite element (MSFE) method by Hou and Wu (1997), the multiscale finite volume method (MSFV) by Jenny et al. (2003), the multiscale mixed finite element method (MSMFE) by Chen and Hou (2003) and Aarnes (2004), the two-scale conservative subgrid approach by Arbogast (2002) and many others. Generally in most of these methods, by imposing different gridding on the global domain (coarse and underlying fine) a set of equations is derived for coarse-scale flow calculations and the underlying fine grid. Fine grid equations are locally defined partial differential equations which are imposed with special reduced boundary conditions. The solution of these problems leads to definition and calculation of basis functions. These functions are used in linear combination to reconstruct fine-scale global solution. These functions must also be frequently updated to incorporate multiphase flow mobility-dependent variables in upscaling. For maintaining the computational efficiency, adaptive schemes are implemented in multiscale methods. The adaptivity relies on the fact that the reservoir processes are phenomenologically highly local in space and time, so that there is a potential to switch back efficiently to fine scale only where and when needed.

As an approximate alternative to multiscale methods, we can implement a post-processing downscaling step into an upscaling method to reconstruct fine-scale properties. This step is carried out by a numerical procedure performed on coarse velocities and coarse pressure. In this study, we use an improved approach of nested-gridding (NG) downscaling within a dual mesh framework (Gautier et al. 1999; Audigane and Blunt 2004). We couple this proposed modified version of downscaling with adaptive local-global (ALG) upscaling. The modification in NG which is the main aspect of this study is attributed to imposing a more actual boundary condition assumption for velocity reconstruction. This is achieved by using local velocity fields obtained from extended regions in upscaling stage. Furthermore, to obtain a fine velocity distribution from coarse scale we propose a methodology that utilizes velocity basis functions. These functions are calculated and stored at initial time to be reused for the rest of simulation.

In the line of application of upscaling-downscaling technique, Kippe et al. (2008) presented a complete comparison of coupled technique of ALG-NG algorithm with three most common multiscale methods. They concluded that ALG-NG is a robust method with a higher computational overhead compared to multiscale methods. However, it is much simpler to set up and downscaling can fit into any upscaling algorithm. The purpose of this study, away from computational concerns, remains in enhancements of downscaling by suggested modifications in NG approximation.

Outline This article is laid out as follows. In Sect.2, we briefly describe the governing equations and single-phase and multi-phase upscaling problems. In Sect. 3, the fundamentals 
of upscaling-downscaling technique will be presented. It is followed by presentation of suggested modifications as the main aspects of this article. Next, in Sect.4, the numerical examples, results, and comparison of methods are given. For a study on computational efficiency, in Sect.5, we perform a three-dimensional waterflooding simulation with different methods. Finally, we summarize the article with conclusions and suggestions in Sect.6.

\section{Definition of Upscaling Problems}

The main problem of upscaling arises from the computational expense of solving for the pressure equation which often has a strong variation in its coefficients, mainly due to geological heterogeneity in absolute permeability. The pressure equation in compact form in case of no-gravity no-capillary multiphase incompressible and immiscible flow in domain $\Omega$ is:

$$
\nabla \cdot \mathbf{v}_{\mathrm{t}}=q \text { and } \mathbf{v}_{\mathrm{t}}=-\mathbf{k} \lambda_{\mathrm{t}} \nabla p \rightarrow-\nabla \cdot\left[\mathbf{k} \lambda_{\mathrm{t}} \nabla p\right]=q, \text { in } \Omega,
$$

where $q$ represents source and sink and contributions at the boundaries and $\mathbf{v}_{\mathrm{t}}$ is the total Darcy velocity of the fluid, for example for a waterflooding in an initially oil-saturated reservoir we have:

$$
\mathbf{v}_{\mathrm{t}}=\mathbf{v}_{\mathrm{o}}+\mathbf{v}_{\mathrm{w}} \text {. }
$$

The total velocity is related to pressure gradient $\nabla p$, absolute permeability, $k$, and in case of multiphase flow, total mobility, $\lambda_{\mathrm{t}}=\lambda_{\mathrm{o}}+\lambda_{\mathrm{w}}, \lambda_{l}=k_{\mathrm{r} l}\left(S_{l}\right) / \mu_{l}$, which $k_{\mathrm{r} l}, S_{l}$, and $\mu_{l}$ are, respectively, relative permeability, saturation, and viscosity of phase $l$. For a system of $N$ cells the discretized model leads to an $N \times N$ banded matrix for finite difference representation. Inverting this matrix is usually the most time-consuming part of the reservoir simulation. Efficient solvers have been developed to improve computational speed. The main focus of the upscaling studies, in parallel to the development of solvers, is to reduce computation costs of solution of this equation. The methods that aim to do so are generally referred to as pressure equation upscaling.

After computation of the pressure and total velocity, the saturation of phase $l$ is solved by the saturation equation:

$$
\phi \frac{\partial S_{l}}{\partial t}+\nabla \cdot\left[f\left(S_{l}\right) \mathbf{v}_{\mathrm{t}}\right]=q_{l}, \quad \text { in } \Omega
$$

where $\phi$ is the porosity, $q_{l}$ is the contribution of phase $l$ in source and sinks and $f\left(S_{l}\right)$ is the fractional flow function of phase $l$ which without capillarity and gravity is $f\left(S_{l}\right)=\lambda_{l}\left(S_{l}\right) / \lambda_{\mathrm{t}}$. The efforts to reduce the computations for solving the saturation equation are referred to as saturation or transport equation upscaling. This area is beyond the scope of this study. Nonetheless, we refer to efforts to add extra terms to coarse-scale saturation equation to account for sub-grid flow based on the reconstructed velocity (Efendiev and Durlofsky 2003). Further, numerical approaches to solve this equation at fine scale efficiently are a block-based overlapping Schwartz technique (Lee et al. 2008) and streamline solvers (Batycky et al. 1997).

For pressure equation upscaling, one approach to reduce the computations is to find upscaled permeabilities to have equations with fewer degrees of freedom to solve. A common expression for an upscaled permeability or the block equivalent permeability for coarse gridblock $E$, denoted henceforth by $\mathbf{k}^{*}$, is given by: 


$$
\int_{E} \mathbf{k} \nabla p \mathrm{~d} x=\mathbf{k}^{*} \int_{E} \nabla p \mathrm{~d} x .
$$

This equation can either be solved numerically or in special cases it has exact solution. A numerical method commonly referred to as pressure solver method (PSM) (Begg et al. 1989; Christie 1996) is to obtain and invert the solution of fine pressure calculated locally over the domain of coarse-gridblock $E \in \Omega$. The solution is dependent on the choice of boundary condition. One common choice is assuming a generic axis-oriented boundary condition (two sides with a prescribed pressure gradient and two sides with no flow). For example if $x$ is the direction of pressure gradient, we can compute total flow rate $\left(q_{x}^{\mathrm{c}}\right)$ by summation of outlet fluxes. Then $\mathbf{k}^{*}$ in $x$ direction is:

$$
\mathbf{k}_{x}^{*}=-\frac{q_{x}^{\mathrm{c}} \cdot \Delta x}{A \Delta p},
$$

where $\Delta p$ is the assumed pressure gradient, $\Delta x$ is the thickness of the gridblock in $x$ direction, $A$ is the area from which the outlet fluxes exit. By alternating the boundary condition over the sides, we have $\mathbf{k}^{*}$ for other directions. We use this method as a base for conventional upscaling techniques. The technique used here yields a diagonal upscaled permeability tensor and ignores the cross terms. Elsewhere (Durlofsky 1991; Pickup et al. 1994) by application of different boundary conditions and processing of the fine grid solution, the calculation of a full tensor $\mathbf{k}^{*}$ is given.

The directional PSM method, due to an arbitrary and unrealistic boundary condition assumption is prone to errors. This will be addressed in the next section.

Another issue for PSM is the case of multiphase flow upscaling. The PSM technique is valid for single-phase flow or tracer flow where total mobility is constant. In multiphase, however, the existence of $\lambda_{\mathrm{t}}(\mathbf{x}, S)$ as a strong function of saturation creates problems as in each time step the values of saturation changes, which leads to change in $\mathbf{k} \lambda_{\mathrm{t}}(S)$. For this case one approach is to define dynamic pseudo-functions, for each coarse block for saturation to match a global fine-scale solution (Kyte and Berry 1975; Stone 1991; Barker and Thibeau 1997). The match leads to derivation of functional relationships for pseudo-relative permeability, $k_{\mathrm{r}}^{*}$, versus volume average saturation $\left(\bar{S}_{i}\right)$ of a coarse block $E_{i}$. This can be written as: $-\nabla \cdot\left(\lambda_{\mathrm{t}, i}^{*}\left(\bar{S}_{i}\right) \mathbf{k}^{*} \nabla p^{\mathrm{c}}\right)=q^{\mathrm{c}}$ where $p^{\mathrm{c}}$ and $q^{\mathrm{c}}$ denote coarse pressure and flux. Unfortunately this method has several drawbacks. It is computationally expensive and dependent on flow conditions (Aarnes and Efendiev 2006). In other words pseudo-functions may only be valid for the exact conditions for which they were derived. Alternatively, we consider the effects of mobility change within the framework of transmissibility upscaling. More description about transmissibility upscaling will also be given in the next section.

\section{Upscaling-Downscaling Scheme}

In this section, we describe ALG-NG upscaling-downscaling technique and the modifications implemented to enhance quality of downscaling. The improvements are based on more detailed information obtained in the upscaling stage. Also we utilize basis functions for downscaling and prolongation of coarse-scale velocities to original grid. The upscalingdownscaling method that we use is introduced in Chen et al. (2003) and Chen and Durlofsky (2006) and later modified by Kippe et al. (2008). In the context of an upscaling-downscaling algorithm the numerical dissipation error of coarse-scale saturation is removed by solving the saturation in the scale of the original fine grid. 
Fig. 1 An illustration of application of global information in terms of surrounding coarse pressure that defines boundary conditions for local solvers in transmissibility upscaling. The gray square is the extended region or jacket around coarse target block and thick rectangles represent the area in which volume average pressures are calculated for upscaling

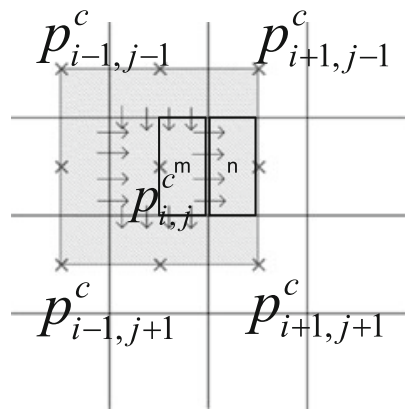

We describe ALG upscaling and the proposed modifications in NG downscaling in the following subsections.

\subsection{ALG Upscaling}

Local-global upscaling is performed by an iteration loop to correct the assumed boundary condition used to compute upscaled values. Here, we use a version in which the correction is performed to upscale transmissibilities. In a finite difference discretization, the transmissibility, $T_{i j}$, based on the two-point flux approximation (TPFA) over an interface $\gamma_{i j}$ is defined as:

$$
T_{i j}=2\left|\gamma_{i j}\right|\left(\frac{\Delta x_{i}}{\lambda_{i}}+\frac{\Delta x_{j}}{\lambda_{j}}\right)^{-1}, \sum_{j} T_{i j}\left(p_{i}-p_{j}\right)=-\int_{E_{i}} q \mathrm{~d} x,
$$

where $\lambda=\mathbf{k} \lambda_{t} . T_{i j}$ relates the flow from one block, $i$, to an adjacent block, $j$, in terms of the pressure difference between the blocks. The sum in Eq. 6 is taken over all non-degenerate interfaces, i.e., over all $i$ such that $\partial E_{j} \cap \partial E_{i}$ has a positive measure. For coarse scale, again under the assumption of TPFA, the upscaled transmissibilities, denoted henceforth by $T^{*}$ of the interface between two coarse blocks, can be computed from the local or extended local problems (hashed area in Fig.1). The solution of these local problems provides the values of upscaled transmissibilities by:

$$
T_{m, n}^{*}=\frac{q_{m, n}^{\mathrm{c}}}{\langle p\rangle_{m}-\langle p\rangle_{n}}
$$

where $q_{m, n}^{\mathrm{c}}=-\int_{\Gamma_{m, n}}\left(\mathbf{k} \lambda_{t}(S) \nabla p\right) \cdot \mathbf{n d} l$ is the total flux obtained by summation of fine grid flow (shown as arrows in Fig.1) across the coarse block interface, $\Gamma_{m, n}$, between two coarse blocks that contain sub-blocks $m$ and $n$. The quantities $\langle p\rangle_{m}=\frac{1}{\left|E_{m}\right|} \int_{E_{m}} \nabla p \mathrm{~d} x$ and $\langle p\rangle_{n}=\frac{1}{\left|E_{n}\right|} \int_{E_{n}} \nabla p \mathrm{~d} x$ are the volume averages of the fine-scale pressure over sub-blocks $E_{m} \subset E_{i, j}$ and $E_{n} \subset E_{i \pm 1, j \pm 1}$, respectively. In the next iteration, the values of surrounding coarse-scale pressures $p_{i \pm 1, j \pm 1}^{\mathrm{c}}$ (or global solution) are used to improve the boundary conditions in local upscaling. With new values of upscaled transmissibility a new iteration is carried out to reach a consistent pressure field and transmissibility set.

The transmissibility upscaling avoids the secondary error otherwise encountered in calculation of coarse transmissibilities based on upscaled permeabilities (Chen et al. 2003). However, $q_{m, n}^{\mathrm{c}},\langle p\rangle_{m}$ and $\langle p\rangle_{n}$ in a multiphase case, for each local equation vary with time due to change in total mobility values. This implies that we have to update the upscaled values 
in each time step of simulation. The updating makes this method applicable to multiphase flow and supersedes the need to define pseudo-relative permeability functions.

The correction to the boundary conditions of the local problem is vital to take into account the effects of non-local complexities within the porous medium that stretch beyond the domain of a local coarse block. The derivation of boundary conditions from global flow is rather a nontrivial task. Here, for two dimensions, we use a bilinear interpolation of coarse pressures values, $p_{i \pm 1, j \pm 1}^{\mathrm{c}}$, to provide the local Dirichlet boundary conditions. Further, it should be noted that the global information obtained by solving coarse-scale problem depends on flow scenarios. In Chen et al. (2003), authors employed directional generic axes-oriented flows, while in later version in Chen and Durlofsky (2006), the global information is derived from specific flow scenario. This choice provides coarse-scale parameters that are adapted for any type of global flow. Here, we use this ALG upscaling version denoted by ALG.

In ALG upscaling, besides adaptation to specific flow, a thresholding technique for maintaining computational efficiency is employed. That is, in multiphase simulation, after computation of a set of upscaled transmissibilities in initial time step, for the next time step, only a portion of transmissibilities are recomputed. Hence, the recomputation is performed only for the coarse blocks that their total mobilities change significantly. Here, the criterion to detect these coarse blocks is taken from Jenny et al. (2005):

$$
\frac{1}{1+\epsilon_{\lambda}}<\frac{\lambda_{t, i}^{n+1}(S)}{\lambda_{t, i}^{n}(S)}<1+\epsilon_{\lambda}
$$

where $\lambda_{t, i}(S)$ denotes total mobility of coarse block $i$ and $\epsilon_{\lambda}>0$ is a user-defined threshold value. If any coarse block violates the inequality of Eq. 8 , the transmissibilities of that block must be recomputed. An advantage of enforcing Eq. 8 besides computational gain is effective elimination of the number of anomalous coarse-scale properties. Anomalous transmissibility values are encountered when global information (interpolated coarse-scale pressure) is not consistent with local regions with low flow rates (Chen and Durlofsky 2006). This inconsistency is seen when the coarse-scale pressures lead to very small pressure difference $\left(\Delta p^{\mathrm{c}}\right)$. If the flow rate $\left(q^{\mathrm{c}}\right)$ is also very small, there can be sign difference between these two values, so the calculated transmissibility will be negative. If $\Delta p^{\mathrm{c}}$ is considerably smaller than $q^{\mathrm{c}}$, the calculated transmissibility in Eq. 7 becomes anomalously large. By enforcing threshold, the recalculation of transmissibilities in these low flow regions is avoided.

\subsection{Modified NG Downscaling}

To reduce the errors of representing saturation at coarse scale, a downscaling of velocities from coarse solution to fine grid is performed. In this technique, we solve for the pressure distribution in the upscaled grid and include the fine-scale heterogeneities in the computation of the phase saturations. In other words, we solve the saturation at a grid nested inside the coarse grid, hence the name, $N G$. Other common terminology used is simulation on a dual mesh framework.

Dual mesh method was first used by Ramé and Killough (1992) to model a single-phase miscible flow. They used a finite element method to solve the pressure field on the coarse scale. This solution is then projected by spline interpolation procedure to the fine grid. A finite difference scheme was used for the saturation equation that was solved on the fine grid. Meanwhile, a restriction operation is performed periodically to rescale the coefficient matrix for the discretized pressure equation on the coarse grid. This operation will inform the coarse grid about the total mobility changes occurring at fine grid. 
Fig. 2 An illustration of downscaling: distributing coarse scale velocity values (bigger arrows) into fine-scale velocities (smaller arrows) as Neumann boundary condition

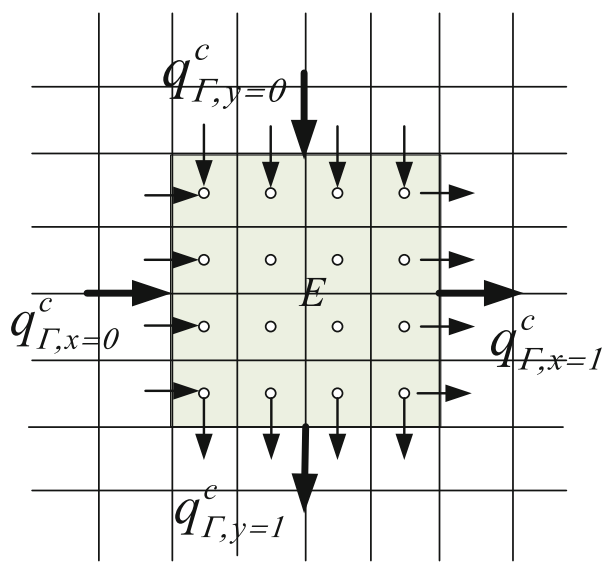

To avoid the artifacts of a non-physical interpolation, Guérillot and Verdière (1995) introduced a dual mesh method where the velocity field was reconstructed within each coarsegridblock by solving the pressure using approximate boundary conditions. For the boundary conditions Gautier et al. (1999) assumed constant fine fluxes across each coarse face, while Chen et al. (2003) and Audigane and Blunt (2004) used fine-scale inter-block transmissibility. In this case, as shown in Fig.2, the main equation for reconstruction of velocity in each coarse gridblock is:

$$
\begin{aligned}
\mathbf{v}_{E} & =-\mathbf{k}_{E} \nabla p, \quad \nabla \cdot \mathbf{v}_{E}=q \text { in } E, \\
\left.\mathbf{v}_{E} \cdot \mathbf{n}\right|_{\Gamma} & =q_{\Gamma}^{\mathrm{c}} \cdot \frac{t_{\gamma i}}{\sum_{\gamma i \subset \Gamma} t_{\gamma i}} \text { on } \partial E,
\end{aligned}
$$

where $\mathbf{v}_{E}$ is the total velocity field for coarse gridblock, $E ; q_{\Gamma}^{\mathrm{c}}$ is the coarse-scale flux across the coarse edge, $\Gamma$, calculated from upscaling stage, and $t_{\gamma i}$ is the fine-scale transmissibility of interface, $\gamma_{i} \subset \Gamma$. We have Nc (number of coarse gridblocks) such equations to solve. Then a global reconstructed velocity field is obtained simply by union of the patches of velocities:

$$
\mathbf{v}^{\mathrm{rec}}=\bigcup_{j=1}^{\mathrm{Nc}} \mathbf{v}_{E j} \quad \text { in } \Omega .
$$

The vector $t_{\gamma i} /\left(\sum_{\gamma i \subset \Gamma} t_{\gamma i}\right)$ in Eq. 9 is in fact a partitioning vector for attributing high permeable boundary fine cells a higher share of coarse flow and similarly lower shares for less impermeable cells. This proportionality is merely local judgment and is prone to errors in cases that a high permeable single fine cell is unconnected to high flow paths. To enhance the proportionality, we try to use a more actual partitioning vector. Hence, we change the proportion to $\left|v_{\gamma_{i}}\right| /\left(\sum_{\gamma i \subset \Gamma}\left|v_{\gamma_{i}}\right|\right)$, where $v_{\gamma_{i}}$ is the fine-scale velocity over interface $\gamma_{i}$. This change obviously requires the original fine velocity distribution. As an approximate alternative, we use distributions obtained by solving for extended local problems in local-global upscaling stage. These solutions provide sufficiently wide velocity fields over the extended region to detect a better picture for connection of flow paths. The indices for prolongation can be easily calculated once for an incompressible case in the first iteration of local-global upscaling. In this case, we change Eq. 9 to: 
Fig. 3 Illustration of the decomposition of NG problem in two dimensions into four equations that determine velocity distribution basis functions
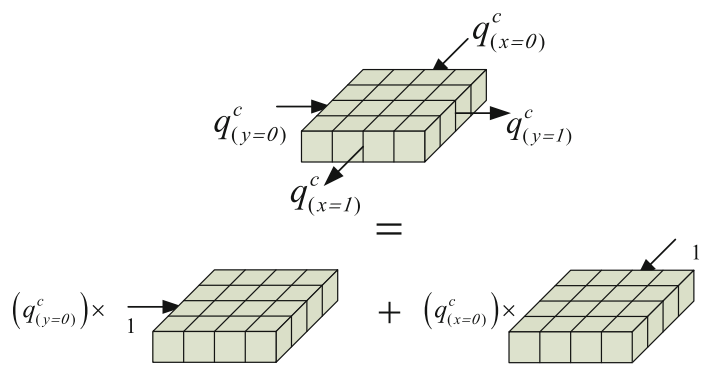

$+$

$\left(q_{(y=l)}^{c}\right) \times$

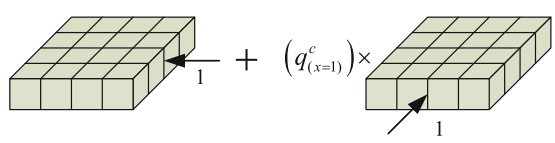

$$
\mathbf{v}_{E}=-\mathbf{k}_{E} \nabla p, \quad \nabla \cdot \mathbf{v}_{E}=q \text { in } E,
$$$$
\left.\mathbf{v}_{E} \cdot \mathbf{n}\right|_{\Gamma}=q_{\Gamma}^{\mathrm{c}} \cdot \frac{\left|v_{\gamma_{i}}^{\mathrm{app}}\right|}{\sum_{\gamma i \subset \Gamma}\left|v_{\gamma_{i}}^{\mathrm{app} \cdot}\right|} \text { on } \partial E \text {, }
$$

where $\mathbf{v}^{\text {app. }}=v_{\gamma_{i}}^{\text {app. }}$ represents an approximated velocity field from local-global upscaling stage. We denote this modification as MNG.

The second suggestion is to improve the computational efficiency. This is achieved by elimination of the constraint to reconstruct the fine pressure distribution. In Gautier et al. (1999), the central fine gridblock is assumed known and equal to the pressure of the coarse gridblock. The specification of pressure in this technique is necessary to make Eq. 9 solvable. However, in our practice, the reconstructed pressure field contains several peculiarities and is not of a comparable quality to the fine reference model.

If we neglect the emphasis of having a fine-scale pressure distribution, instead we can try only to reconstruct a mass conservative velocity field. This allows us to decompose the problem into four equations in two dimensions (as shown in Fig. 3) and six in three dimensions (number of coarse edges) plus particular solutions for wells. The solvability in this case is achieved by an arbitrary fixation of pressure for one cell, e.g., equating the pressure of the first cell to the pressure of the coarse gridblock $1\left(p_{1}=P_{\mathrm{c}}\right)$. The pressure solution, in this fashion, is non-physical but its gradient provides useful velocity distributions.

For each decomposed equation, we impose Neumann boundary condition, derived from partitioning of a unit-value coarse-scale flux over the corresponding coarse edge. For example for a coarse gridblock, $E$, we compute a distribution function denoted by $\mathbf{v}_{\Gamma i}$ for edge $\Gamma i \subset \partial E$, obtained by boundary condition assumed as $\left.\mathbf{v}_{\Gamma i} \cdot \mathbf{n}\right|_{\Gamma i}=1 \times\left(\left|v_{\gamma_{i}}^{\text {app. }}\right| / \sum_{\gamma i \subset \Gamma}\left|v_{\gamma_{i}}^{\text {app. }}\right|\right)$ on $\Gamma_{i}$ and open flow over the other edges. Henceforth, we refer to these distributions as basis functions.

After calculation of the distribution basis functions, we can generate the same velocity distribution of Eq. 10 by means of a linear superposition for coarse gridblocks and a union for the global domain:

$$
\mathbf{v}^{\mathrm{rec}}=\bigcup_{j=1}^{\mathrm{Nc}}\left(\sum_{i=1}^{M} q_{\Gamma i}^{\mathrm{c}} \cdot \mathbf{v}_{\Gamma i}\right)_{j}+\mathbf{v}_{\mathrm{p}}, \quad \text { in } \Omega,
$$




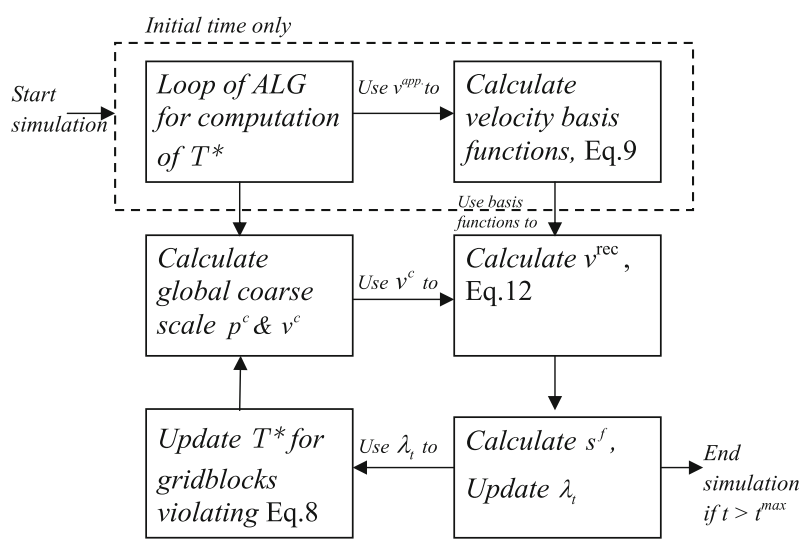

Fig. 4 Flowchart for upscaling-downscaling with use of basis function

where Nc is number of coarse gridblocks, $M$ is space dimension, $q_{\Gamma i}^{\mathrm{c}}$ is coarse velocity over coarse edge, $\Gamma i \subset \partial E$, and $\mathbf{v}_{\mathrm{p}}$ is a particular solution only for well coarse gridblocks calculated as:

$$
\mathbf{v}_{\mathrm{p}}=-\mathbf{k}_{E} \nabla p, \quad \nabla \cdot \mathbf{v}_{\mathrm{p}}=q \quad \text { on } \quad E_{\mathrm{well}}
$$

The calculation of $\mathbf{v}_{\Gamma i}$ and $\mathbf{v}_{\mathrm{p}}$ are performed only in initial time step so that the downscaling does not require the solutions of Eq. 9 for the rest of simulation, furthermore, such calculations are obviously parallelizable. These factors can be exploited for computational gains. Moreover, aiming only at constructing a conservative velocity field, different global boundary conditions can be simply incorporated into this framework. The flowchart of application of this technique is given in Fig. 4.

Use of the basis functions to reconstruct fine-scale distributions has also been examined in the context of MSFV and MSMFE. In MSFV (Jenny et al. 2003) the pressure basis functions are used to derive effective upscaled transmissibilities. For fine-scale velocity reconstruction, this algorithm uses 9 local problems (in 2D) and 27 local problems (in 3D) to construct and store a secondary set of functions as velocity basis function. Also, in MSMFE (Aarnes 2004) an additional set of basis functions for velocity are considered in addition to the usual basis functions for pressure considered in conventional finite element methods. The role of these basis functions is similarly to incorporate the effect of total mobility change and underlying subgrid heterogeneities.

In the line of application of a static velocity field (obtained only at initial time), Aarnes and Efendiev (2006) used homogenization theory to prove that under some assumptions, the two-phase flow velocity, can be approximated by a static part that does not depend on saturation, times a time dependent function in each coarse block. Also Khoozan et al. (2011) replaces the time-consuming reconstruction step in the dual mesh method with a fast analytical solution. However, in deriving the analytical solution, the subgrid heterogeneity of each coarse gridblock is replaced with homogeneous upscaled value. This can be an undermining factor. Instead we use numerical solution for deriving the static part and the coarse velocity field as weights to compute the global reconstructed velocity field. In the next section, we will apply and compare this algorithm with fine reference model on different cases of flow simulation and various heterogeneous media and boundary conditions. 
Table 1 Upscaling-downscaling methods used in comparison study

\begin{tabular}{ll}
\hline Method & Description \\
\hline PSM-NG & Conventional NG on PSM \\
PSM-MNG & Modified NG on PSM \\
ALG-NG & Conventional NG on ALG upscaling in single phase \\
ALG-MNG- & Static (with application of basis function) modified NG \\
& on ALG upscaling in multiphase \\
ALG-MNG+ & Dynamic (without application of basis functions) modified NG \\
& on ALG upscaling in multiphase \\
\hline
\end{tabular}

\section{Numerical Experiments}

In this section, we compare the algorithms defined in the previous section with different boundary conditions and over different heterogeneous media. For boundary conditions, we assume a quarter five-spot pattern as well as flow induced by constant injection rate on one end and constant pressure production on the other end. The heterogeneous models include: two- and three-dimensional models with permeability data from Model 2, the 10th SPE Comparative Solution Project (Christie and Blunt 2001), henceforth referred to simply as the SPE10 model, and a synthetic channelized model (Chen et al. 2004). The SPE10 model consists of $60 \times 220 \times 85$ cells. The top 35 layers represent a permeability field with quite a smooth variation in the coefficients. The bottom 50 layers constitute a fluvial formation with narrow high flow channels which makes upscaling difficult.

Two waterflooding scenarios are considered in the following subsections. The first one is a tracer (linear) flow simulation in which the engaging fluids act as a single-phase flow specification. The second scenario is an incompressible waterflooding case which represents multiphase flow case. For each scenario, a range of combination of upscaling and downscaling methods is compared with each other. The description and denotation of these techniques are given in Table 1.

To assess the quality of the reconstructed saturation profiles of various upscaling-downscaling techniques, we compare them to fine-scale reference solutions. This is achieved by using:

$$
\delta\left(s^{\mathrm{rec}}, s^{\mathrm{ref}}, t\right)=\frac{\left\|s^{\mathrm{ref}}(., t)-s^{\mathrm{rec}}(., t)\right\|_{L^{2}}}{\left\|s^{\mathrm{ref}}(., t)\right\|_{L^{2}}},
$$

where $s^{\text {ref }}$ represents reference fine saturation and $s^{\text {rec }}$ represents reconstructed saturation in different methods. Time $(t)$ is expressed by pore volume injected. Similarly, anywhere needed, for coarse-scale pressure error measurement we will use:

$$
\delta\left(P^{\mathrm{c}}, P^{\mathrm{f}}, t\right)=\frac{\left\|P^{\mathrm{f}}(., t)-P^{\mathrm{c}}(., t)\right\|_{L^{2}}}{\left\|P^{\mathrm{f}}(., t)\right\|_{L^{2}}},
$$

where $P^{\mathrm{c}}$ is coarse pressure from various upscaling methods and $P^{\mathrm{f}}$ is volume-averaged pressure from fine-scale reference method.

\subsection{Tracer Flow Case}

In tracer flow, we have an injected fluid which has the same properties as the initial fluid in place. This is equivalent when we set a linear flow function $f(S)$ by viscosity ratio of $M=1$ and relative permeability curves as: 
Layer 10

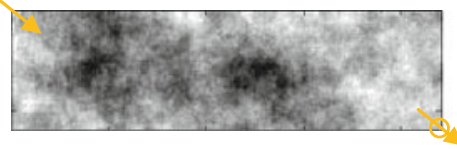

Layer 47

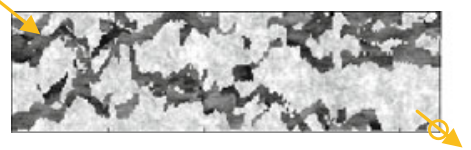

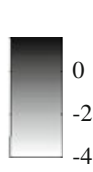

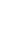

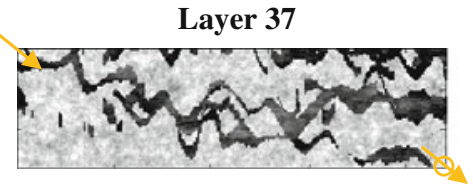

Layer 68

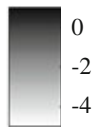

Layer 37

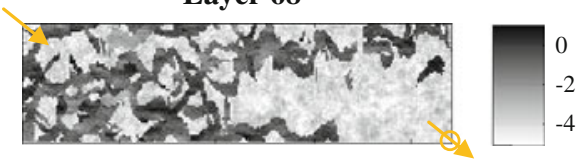

Fig. 5 Logarithm of permeability fields for layers 10, 37, 47, and 68, from Model 2 from the 10th SPE Comparative Solution Project (Christie and Blunt 2001). Inlet and outlet are also shown by arrows for the case of corner-to-corner flow

Table 2 Comparison of average of $\delta\left(s^{\mathrm{rec}}, s^{\mathrm{ref}}, t\right)$ error from different reconstruction schemes for linear flow through layers $10,37,47$, and 68 of SPE10 model

\begin{tabular}{lllll}
\hline Layer & $\begin{array}{l}\text { Average of } \\
\delta\left(s^{\text {rec }}, s^{\text {ref }}, t\right) \text { of } \\
\text { PSM-NG }\end{array}$ & $\begin{array}{l}\text { Average of } \\
\delta\left(s^{\text {rec }}, s^{\text {ref }}, t\right) \text { of } \\
\text { ALG-NG }\end{array}$ & $\begin{array}{l}\text { Average of } \\
\delta\left(s^{\text {rec }}, s^{\text {ref }}, t\right) \text { of } \\
\text { PSM-MNG }\end{array}$ & $\begin{array}{l}\text { Average of } \\
\delta\left(s^{\text {rec }}, s^{\text {ref }}, t\right) \text { of } \\
\text { ALG-MNG }\end{array}$ \\
\hline 10 & 0.1297 & 0.0901 & 0.1285 & 0.0693 \\
37 & 0.4764 & 0.2881 & 0.4710 & 0.2517 \\
47 & 0.6396 & 0.3742 & 0.6153 & 0.2991 \\
68 & 0.4752 & 0.3763 & 0.4727 & 0.2964 \\
\hline
\end{tabular}

$$
k_{\mathrm{rw}}=S \text { and } k_{\mathrm{ro}}=1-S,
$$

where $S$ is the saturation of injected fluid. These assumptions provide a constant total mobility and constant $\mathbf{k} \lambda_{t}$ throughout the simulation. Consequently, the upscaling is a single-phase upscaling. Hence, for the case of ALG upscaling, we do not need to update the upscaled transmissibilities. Also in downscaling stage, we do not have NG- or NG+ schemes, so the comparison is limited to only use of $\mathbf{v}^{\text {app. }}$ or in other words improvements by using MNG compared to NG.

For boundary conditions we assume a source and a sink respectively on top left and bottom right corners of layers 10, 37, 47, and 68 of SPE10 model. Layers 37, 47, and 68 are highly channelized and heterogeneous where the permeability in the system undergoes a wide variation from 0.004 to $20,000 \mathrm{mD}$. Layer 10 is a non-channelized layer. Nonetheless it also shows high variation of permeability. Figure 5 shows the logarithm of permeability for these layers. We assume a constant porosity for the layers and the coarse model is $6 \times 22$ upscaled from original $60 \times 220$ gridcells. For the solution of the saturation equation we implemented a sequential implicit scheme, that is, the pressure equation is first solved, and based on a fixed total velocity, the saturation equation is implicitly solved via an iteration loop in a decoupled sequence.

Table 2 gives the average of $\delta\left(s^{\text {rec }}, s^{\text {ref }}, t\right)$ over 1.5 PVI of simulation. Interestingly, MNG has improved the quality of saturation profiles for ALG better than for PSM. Applied on ALG the improvement by MNG relative to NG at 1.5 PVI for the layers 10, 37, 47 and 68 are respectively $22.19,9.06,22.77$, and $17.01 \%$.

Figure 6 shows water cut curves obtained for different layers at production cell. For layer 10, as we expect from heterogeneity pattern, PSM-NG is within a satisfying range. 

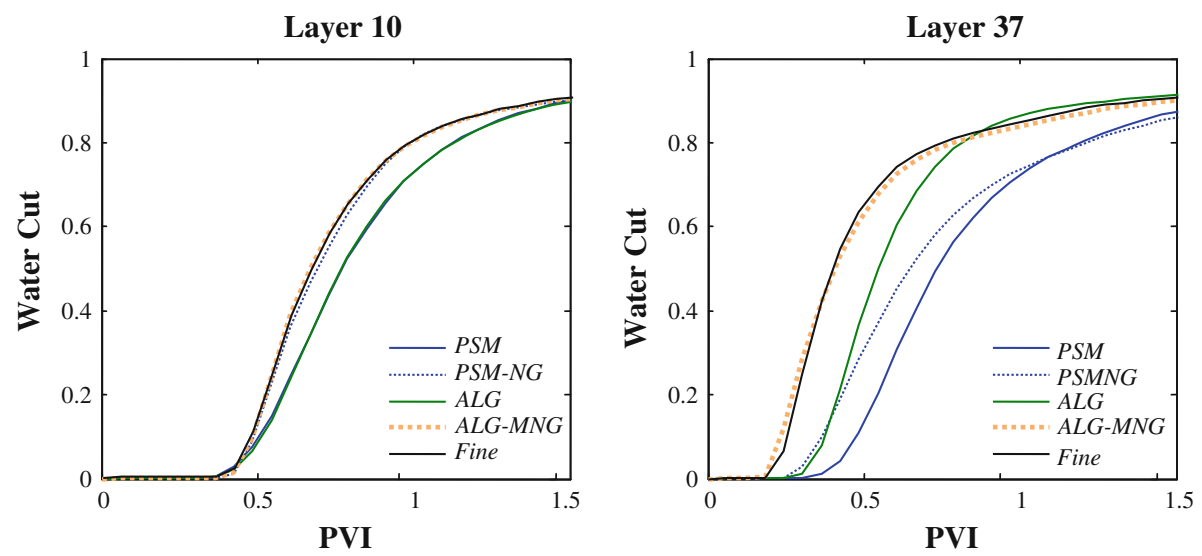

Layer 47

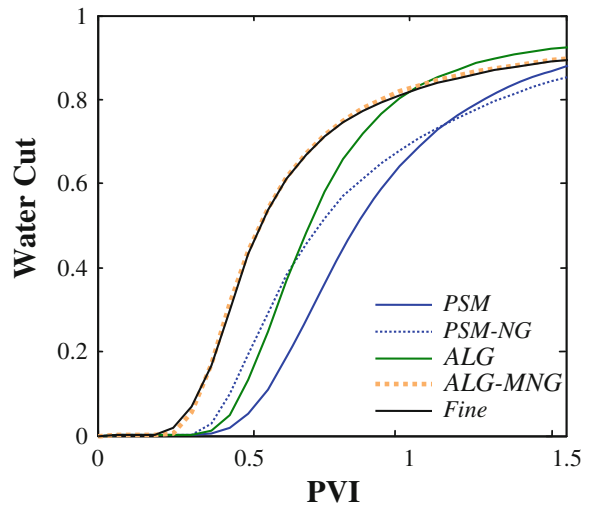

Layer 68

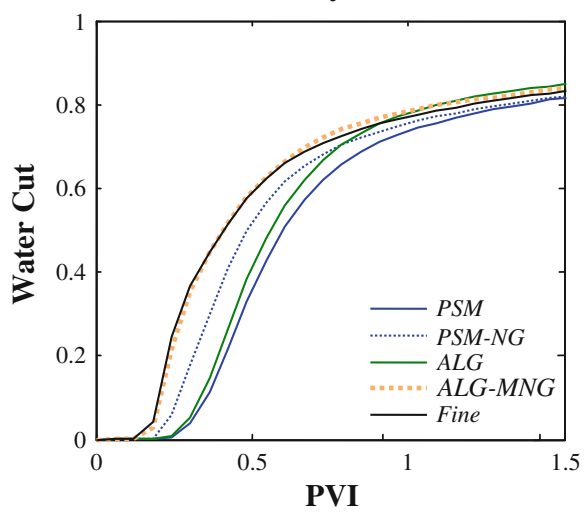

Fig. 6 Water cut curves for linear flow simulations on layers of 10, 37, 47, and 68 for reference fine model and different upscaling and upscaling-downscaling methods

In contrary, the other layers display the importance of corrected transmissibilities by ALG scheme. For these layers the error of homogenization through upscaling exceeds the one from coarse representation of saturation. Therefore, downscaling of velocity field could not remove the error or the discrepancy with fine-scale's curve. We note that the improvement by ALG-MNG compared to ALG-NG is not reflected for the single value of saturation at producing cell that determines the water cut. This pattern occurs for other simulations that will follow.

\subsection{Multiphase Flow Case}

In this section, we test the modifications for a two-phase flow simulation to determine the improvement by MNG compared to NG and investigate the application of MNG- algorithm. For a water flooding, we assume a reservoir initially saturated with oil. Water is injected to displace the oil. For relative permeabilities we set:

$$
k_{\mathrm{rw}}=S^{2} \quad \text { and } \quad k_{\mathrm{ro}}=(1-S)^{2} \text {, }
$$


Layer 10

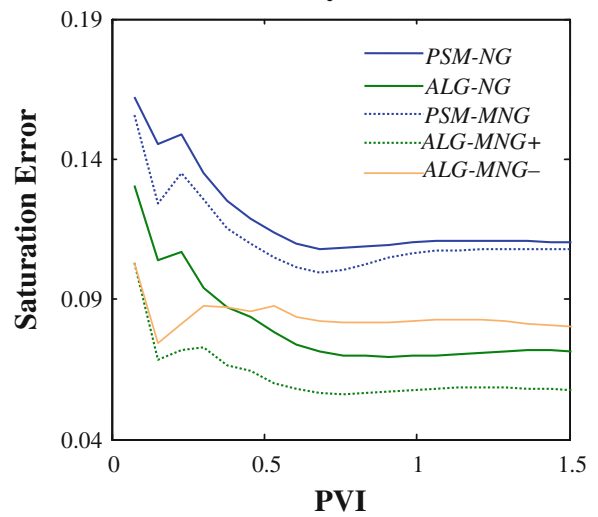

Layer 47

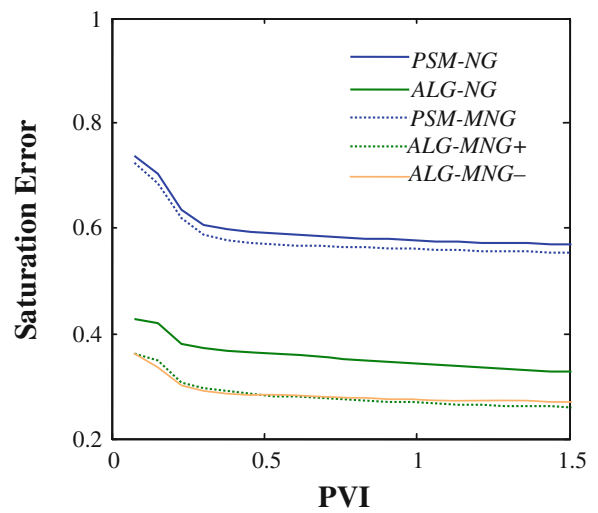

Layer 37

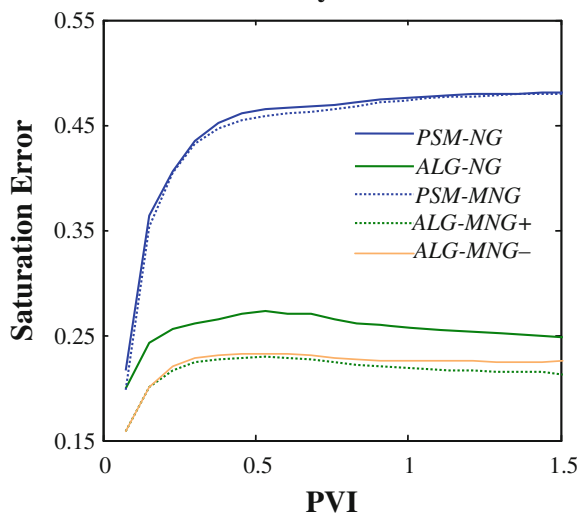

Layer 68

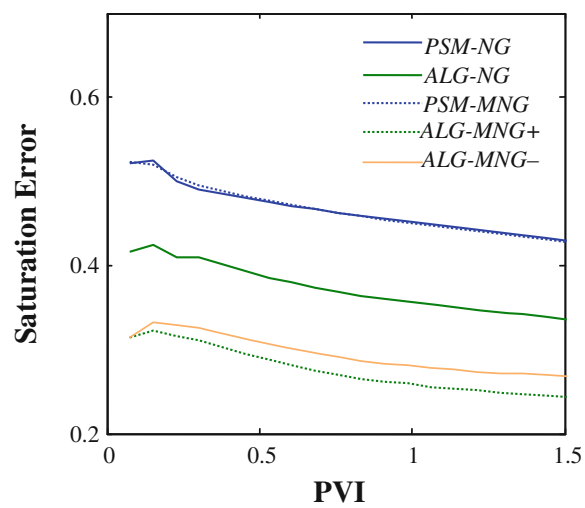

Fig. 7 Comparison of $\delta\left(s^{\text {rec }}, s^{\text {ref }}, t\right)$ error from different reconstruction schemes for multiphase flow $(M=$ 10) through layers $10,37,47$, and 68 of SPE10 model

where $S$ is the saturation of water. Two cases of mobility ratios are examined, $M=\mu_{\mathrm{o}} /$ $\mu_{\mathrm{w}}=0.1$ and $M=\mu_{\mathrm{o}} / \mu_{\mathrm{w}}=10$. For $M=0.1$ the front is sharp and piston-like while for $M=10$, in contrast, the front is smearing and has dispersing protrusions inside the displaced fluid. From a reconstruction point of view, an unstable front (high viscosity ratios) is less prone to create errors in water cut values. The reason is that the increase of water saturation throughout the domain is more gradual due to higher physical smearing and a weak shock. This keeps the change in total mobility small enough that the coarse velocity field can take them into account more effectively. On the other hand, the saturation increase is spatially abrupt and sudden for cases of stable piston-like frontal displacements (low viscosity ratios). This exposes the reconstruction with oscillations and errors because the slightest of differences in locating the front in reconstruction leads to large discrepancy of saturation values measured between different models.

Figure 7 shows the global error measured by $\delta\left(s^{\mathrm{rec}}, s^{\mathrm{ref}}, t\right)$ for different reconstruction algorithms of ALG-NG, ALG-MNG+, ALG-MNG-, and PSM-NG for multiphase flow $M=10$ case. We observe improvement by MNG compared to NG similar to linear flow case. However, the loss of accuracy by ALG-MNG- is visible for layer 10. In contrast, for 
Layer 10- $M=0.1$

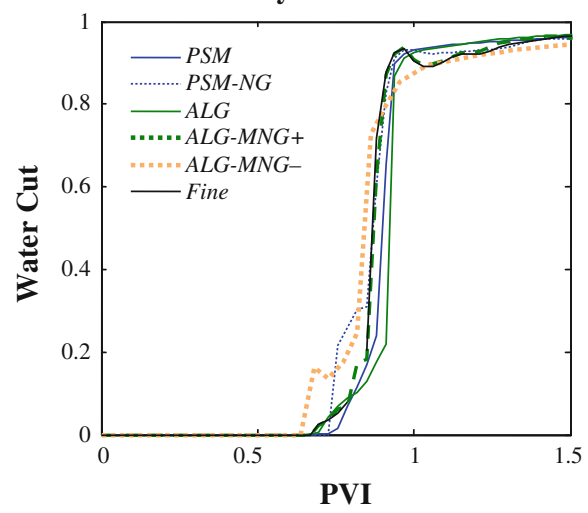

Layer 47- $M=0.1$

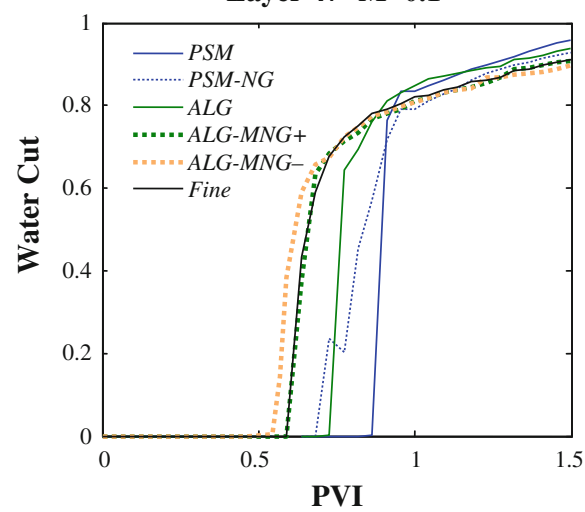

Layer 37- $M=0.1$

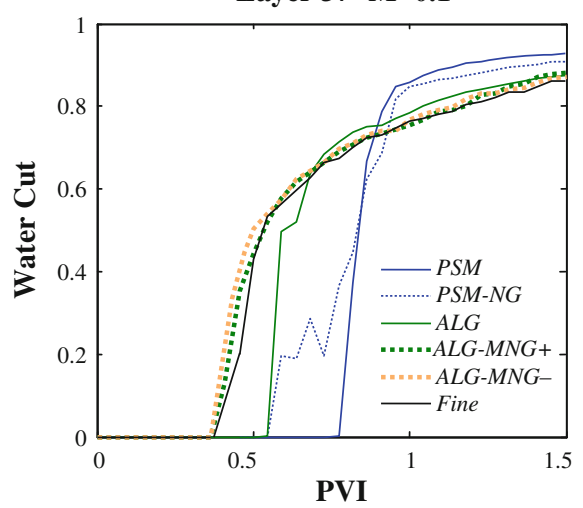

Layer 68- $M=0.1$

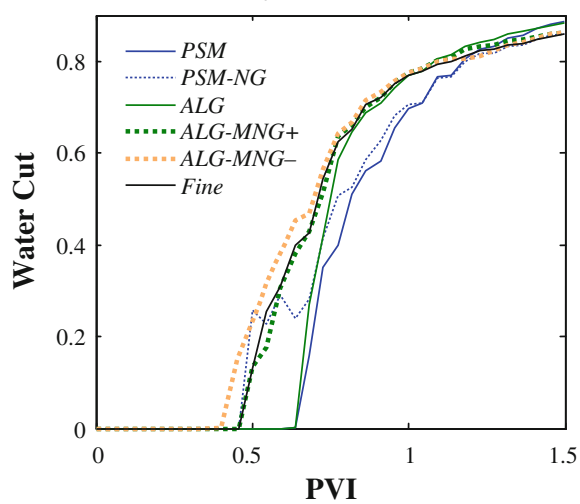

Fig. 8 Water cut curves for multiphase flow simulations $(M=0.1)$ on layers of $10,37,47$, and 68 for reference fine model and different upscaling and upscaling-downscaling methods

channelized layers, the application of ALG is advantageous due to high errors of PSM upscaling. For these layers ALG-MNG+ and ALG-MNG- have almost similar values of error. To conclude, in any case of heterogeneity, MNG is obviously advantageous compared to NG, while for channelized heterogeneity MNG- infers minimal loss of accuracy compared to $\mathrm{MNG}+$.

Figures 8 and 9 show the water cut curves calculated at producing cell for each layer and each viscosity ratio. In each panel, we have compared the curves obtained by ALG-MNGto ALG-MNG+, PSM-NG, and fine reference model. Only we have spotted deterioration of ALG-MNG- curve compared to ALG-MNG+ for case of layer 10 with $M=0.1$, while other cases confirm that non-updated basis functions for multiphase flow produce satisfying results.

Figure 10 shows fine-scale saturation profiles constructed from fine reference model and three upscaling-downscaling algorithms at 1.5 PVI. Obviously, the reconstruction based on PSM suffers significantly from bad upscaled values for coarse velocities due to inaccurate coarse properties and ALG-NG and ALG-MNG- have resulted similar profiles as of the fine-scale reference model. 
Layer 10- $M=10$

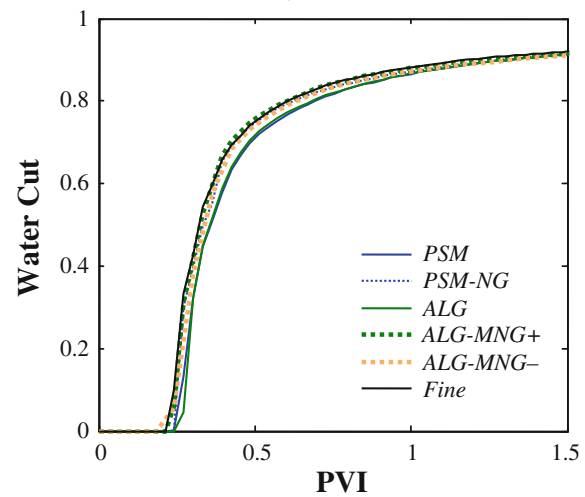

Layer 47- $M=10$

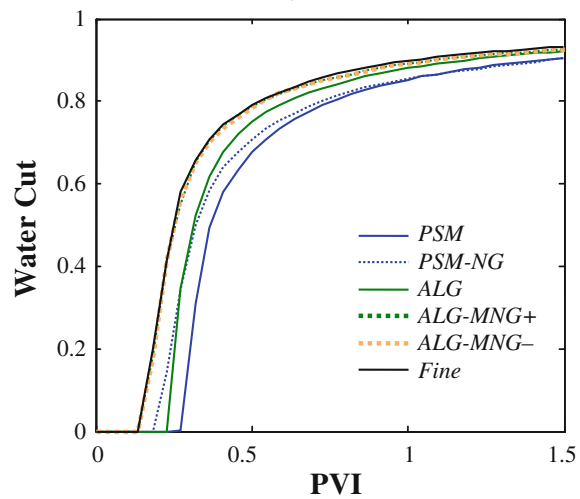

Layer 37- $M=10$

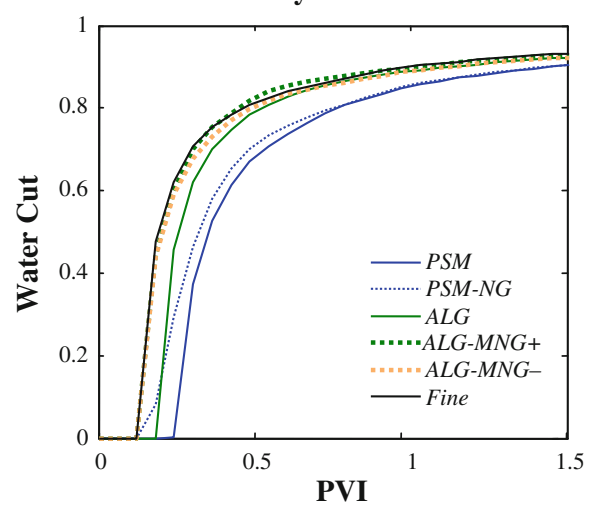

Layer 68- $M=10$

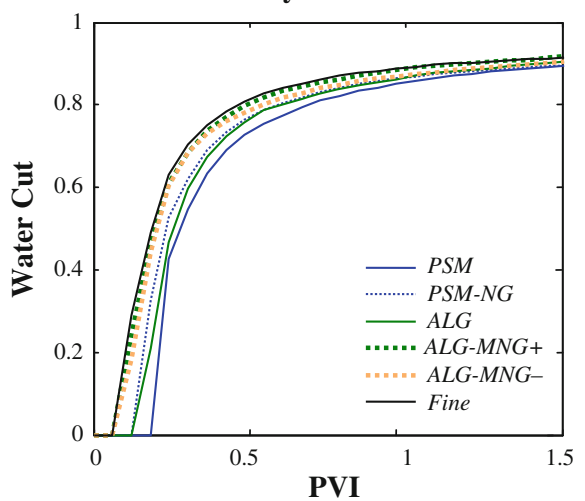

Fig. 9 Water cut curves for multiphase flow simulations $(M=10)$ on layers of 10,37 , 47, and 68 for reference fine model and different upscaling and upscaling-downscaling methods

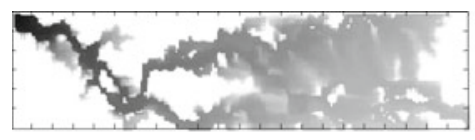

Fine Model

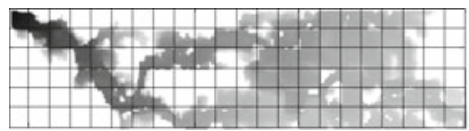

ALG-NG Model
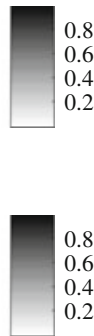

0.2

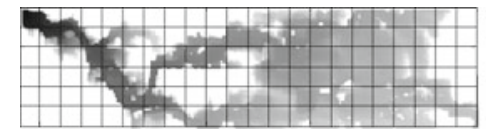

ALG-MNG-Model

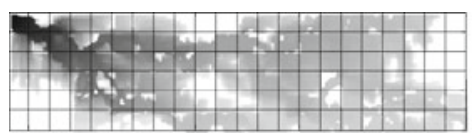

PSM-NG Model

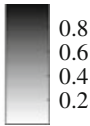

Fig. 10 Fine scale saturation profiles obtained by different method for corner-to-corner flow, layer 47, at 1.5 PVI $(M=10)$

After considering corner-to-corner flow, here, we examine the case of directional flow and constant pressure production. The boundary conditions are set as constant injection rate from one side of the reservoir and a constant pressure production at the other side. Again we upscale the fine layers (layers 37 and 47) from $60 \times 220$ gridcells to $6 \times 22$. Here, only the 
Layer 37

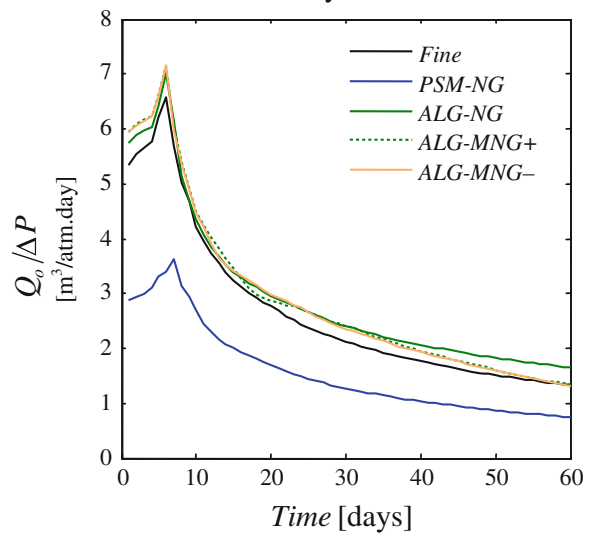

Layer 47

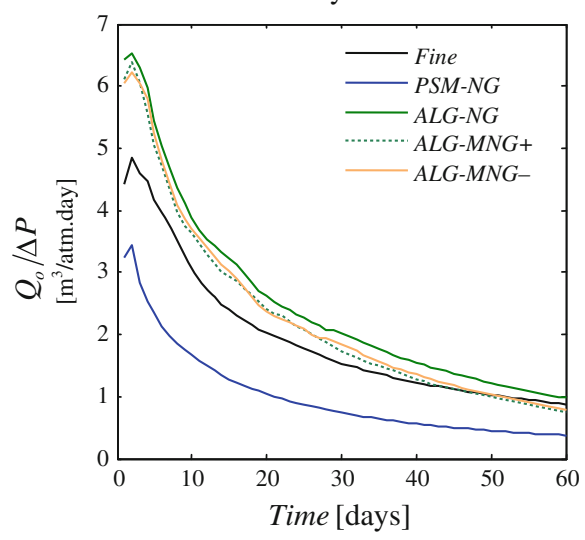

Fig. 11 Comparison of various upscaling-downscaling techniques for directional flow case through $Q_{\mathrm{o}} / \Delta P$

Table 3 Coarse pressure and fine saturation error measurements for directional flow case

\begin{tabular}{llllll}
\hline Model & $\begin{array}{l}\delta\left(P^{\mathrm{c}}, P^{\mathrm{f}}, t\right) \text { of } \\
\text { PSM }\end{array}$ & $\begin{array}{l}\delta\left(P^{\mathrm{c}}, P^{\mathrm{f}}, t\right) \text { of } \\
\text { ALG }\end{array}$ & $\begin{array}{l}\text { Average of } \\
\delta\left(s^{\text {rec }}, s^{\text {ref }}, t\right) \text { of } \\
\text { PSM-MNG }\end{array}$ & $\begin{array}{l}\text { Average of } \\
\delta\left(s^{\text {rec }}, s^{\text {ref }}, t\right) \text { of } \\
\text { ALG-NG }\end{array}$ & $\begin{array}{l}\text { Average of } \\
\delta\left(s^{\text {rec }}, s^{\text {ref }}, t\right) \text { of } \\
\text { ALG-MNG- }\end{array}$ \\
\hline Layer 37 2.1042 & 0.1329 & 0.5553 & 0.3741 & 0.3156 \\
Layer 47 & 0.5096 & 0.0573 & 0.4921 & 0.3408 & 0.2855 \\
\hline
\end{tabular}

viscosity ratio of $M=10$ is considered. For the assessment of the accuracy of the various techniques, we consider the accuracy of the pressure field and oil flow rate by measuring $Q_{\mathrm{o}} / \Delta P$ versus time. In this quantity, $Q_{\mathrm{o}}$ is the fine-scale oil production rate obtained by multiplying the oil fractions of outlet face by total production rate and $\Delta P$ is the average value of the difference between inlet face and the outlet face pressures. The values of $\Delta P$ are computed at coarse scale for upscaling-downscaling techniques as the pressure is not downscaled here.

Figure11 illustrates the improvement of results by ALG-NG, ALG-MNG-, and ALGMNG+ compared to PSM-NG. The improvement is mainly due to better coarse pressure values calculated by ALG upscaling. However, there is only a minimal improvement by ALG-MNG- compared to ALG-NG. Moreover, the application of distribution basis functions is completely reasonable as we observe no deterioration of results by ALG-MNGcompared to ALG-MNG+. In Table 3, the average errors for coarse-scale pressure and finescale saturation are given. These values are calculated respectively by averaging $\delta\left(P^{\mathrm{c}}, P^{\mathrm{f}}, t\right)$ and $\delta\left(s^{\text {rec }}, s^{\text {ref }}, t\right)$ over time $(2 \mathrm{PVI})$.

The next test model, taken from Chen et al. (2004), is one realization of a $2 \mathrm{D}$ synthetic channelized system containing $100 \times 100$ gridcells on a Cartesian coordinate system. This model was generated using multi-point geostatistics (for the channels) and two-point geostatistics (for permeability distributions within each facies). This permeability model (Fig. 12) is characterized by high variations from 0.02 to $6.5 \times 10^{7} \mathrm{mD}$, a mean of $7 \times 10^{4} \mathrm{mD}$ and isotropic normalized correlation lengths varying from 0.05 to 0.5 . In this model, the correlated permeabilities are not aligned with the Cartesian grid lines, but rotated almost at $45^{\circ}$ with respect to the grid. This model represents a case in which the flow solution is highly affected 
Fig. 12 Logarithm of absolute permeability for synthetic channelized model
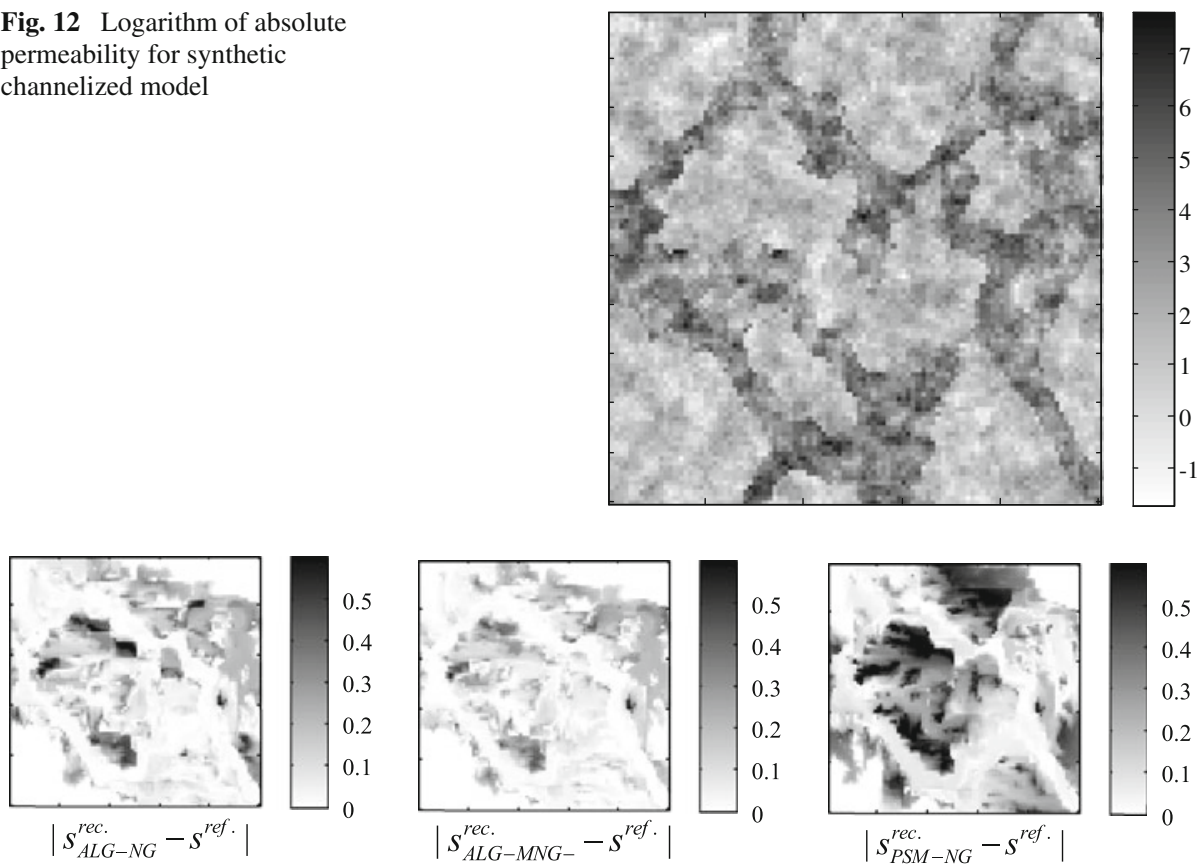

Fig. 13 Improvement in saturation absolute error by modifications in reconstruction of velocity at 2 PVI

by grid orientation and flow conditions in the finite difference scheme, as the preferred flow paths are along high permeabilities, not the model coordinate system. The upscaling is difficult in this case due to high permeability variation, high permeability channel, and complex geometry of heterogeneities.

We examine tracer flow induced by corner-to-corner source and sink configuration. We upscale this model to $10 \times 10$ gridblocks and test the same algorithms of reconstruction as before. In Fig. 13, we have confirmed the performance of ALG-MNG- in lowering the fine-scale saturation error compared to ALG-NG- and PSM-NG.

To investigate the effect of upscaling factor on performance of ALG-MNG-, we have carried different coarsening levels on this test case: upscale from $100 \times 100$ to $25 \times 25$, to $10 \times 10$ and $5 \times 5$. By measuring $\delta\left(s^{\mathrm{rec}}, s^{\mathrm{ref}}, t\right)$, we can see in Fig. 14 that we observe less improvement by MNG compared to NG for $25 \times 25$ case. That is to say for lower upscaling factors (like $25 \times 25$ case with upscaling factor of only 16) proportionality indices calculated by fine-scale transmissibilities are close enough to those calculated by approximate velocity field. In comparison of ALG-MNG- and ALG-MNG+, we observe $\delta\left(s^{\text {rec }}, s^{\text {ref }}, t\right)$ for ALG-MNG- only to exceed that of ALG-MNG+ after 1 PVI for upscaling factors of 400 $(5 \times 5)$ and $100(10 \times 10)$.

In this case, for a 2D upscaling factor of 100 which is a more usual case for reservoir simulation upscaling, we see a good improvement in terms of $\delta\left(s^{\mathrm{rec}}, s^{\text {ref }}, t\right)$ by MNG compared to NG (from 0.3078 to 0.2311 at $2 \mathrm{PVI}$ ) and small deterioration by using MNG- algorithm (from 0.2311 to 0.2584 at $2 \mathrm{PVI}$ ).

In directional flow case, we performed similar analysis of errors for $Q_{\mathrm{o}} / \Delta P$, and errors for coarse-scale pressure and fine-scale saturation. Results are respectively presented in Fig. 15 and Table 4. Similar to layers 37 and 47 that were examined for directional flow, this example shows that high coarse pressure errors by PSM are removed by ALG upscaling. From 


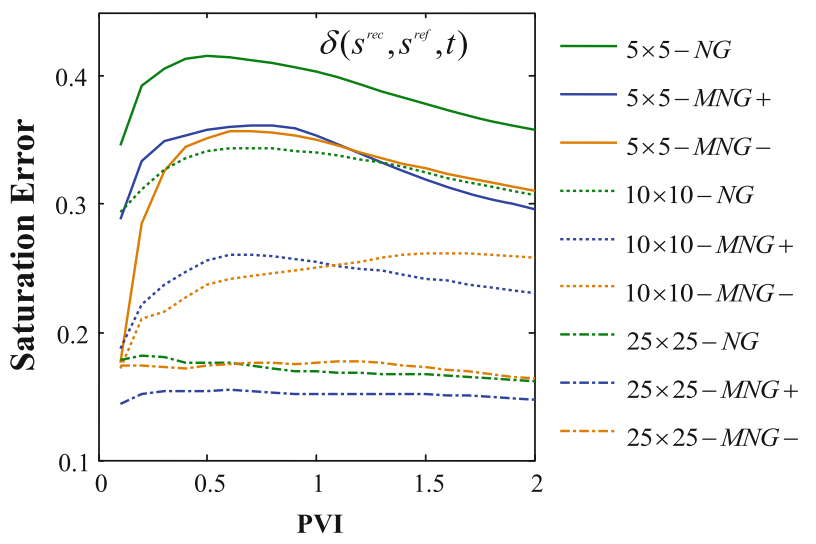

Fig. 14 Effect of upscaling factor on performance of basis functions for downscaling

Fig. 15 Channel case: comparison of various upscaling-downscaling techniques for directional flow case through $Q_{\mathrm{o}} / \Delta P$

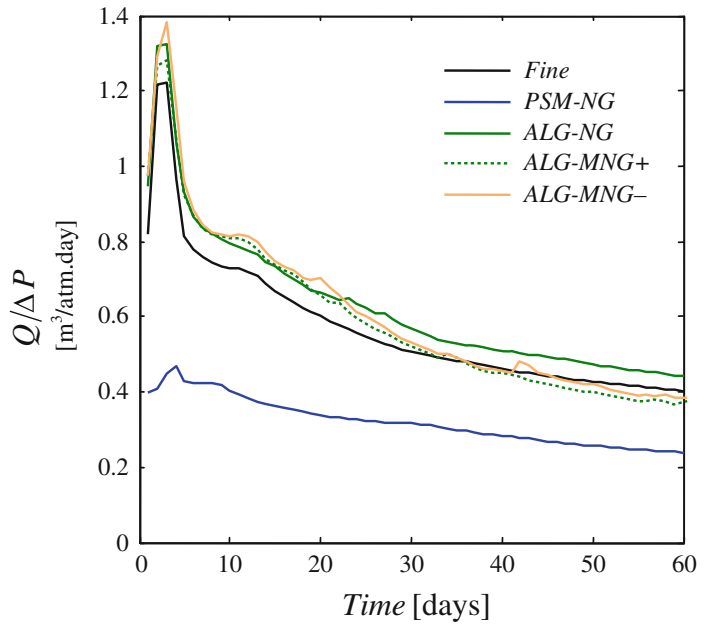

Table 4 Channel case: coarse pressure and fine saturation error measurements for directional flow case at 6PVI

\begin{tabular}{llllll}
\hline Model & $\begin{array}{l}\delta\left(P^{\mathrm{c}}, P^{\mathrm{f}}, t\right) \text { of } \\
\text { PSM }\end{array}$ & $\begin{array}{l}\delta\left(P^{\mathrm{c}}, P^{\mathrm{f}}, t\right) \text { of } \\
\text { ALG }\end{array}$ & $\begin{array}{l}\text { Average of } \\
\delta\left(s^{\mathrm{rec}}, s^{\mathrm{ref}}, t\right) \text { of } \\
\mathrm{PSM}-\mathrm{MNG}\end{array}$ & $\begin{array}{l}\text { Average of } \\
\delta\left(s^{\mathrm{rec}}, s^{\mathrm{ref}}, t\right) \text { of } \\
\text { ALG-NG }\end{array}$ & $\begin{array}{l}\text { Average of } \\
\delta\left(s^{\mathrm{rec}}, s^{\text {ref }}, t\right) \text { of } \\
\text { ALG-MNG- }\end{array}$ \\
\hline Channel case 0.5572 & 0.0988 & 0.3862 & 0.2173 & 0.1720 \\
\hline
\end{tabular}

downscaling point of view, there is some improvements by MNG compared to NG and no underperformance by $\mathrm{MNG}-$ compared to $\mathrm{MNG}+$.

\section{Simulation Runtime Consideration for a 3D Case}

In previous tests, we showed that by the upscaling-downscaling scheme we can generate a good approximation to the flow compared to a fine reference model by ALG-MNG-. 

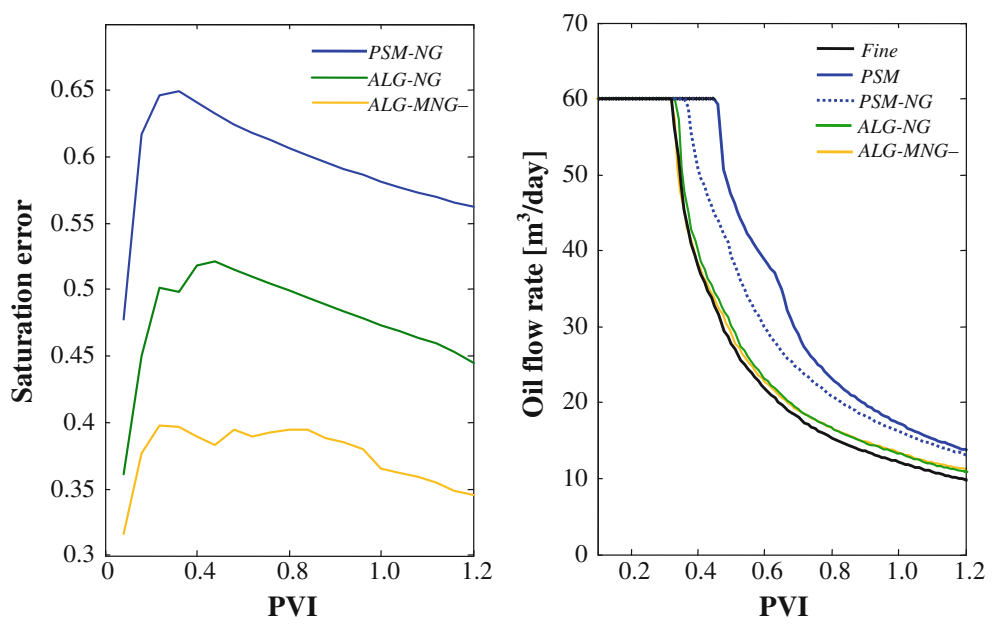

Fig. 16 Left The $\delta\left(s^{\text {rec }}, s^{\text {ref }}, t\right)$ error, right the curves for oil production rate with different methods

However, the most important factor is to keep the efficiency of upscaling in terms of CPU gain and memory saving. In our algorithm, we only focus on pressure equation upscaling and once we reconstruct a velocity field the computation for the saturation equation is as expensive as it is for the fine model. In the calculation of CPU time, we assume that the superposition of velocity basis functions is not computationally demanding as it does not involve any equation to solve. Hence, for the downscaling part the time for NG is not considered. For large-scale reservoir models the superposition is actually a matter of optimized programming and can easily outperform linear solution of local problems.

For a 3D example, we take a section of SPE10 model bottom 50 layers (Upper-Ness fluvial channelized formation). The section is a $60 \times 60 \times 50$ grid with variation of permeability between $9.63 \times 10^{-7}$ to $20 \mathrm{mD}$. We upscale it to $6 \times 6 \times 5$ blocks with PSM, PSM-NG, ALG-NG-, and ALG-MNG- methods as in the previous cases. The water flooding boundary conditions are corner-to-corner flow case with constant pressure production at one corner and constant injection rate at the other. We use a mobility ratio of $M=10$ and relative permeabilities from Eq. 17 for the water and oil phases. For local-global method the computations can be costly given that in three dimensions, calculation of a corrected set of transmissibilities involves extended regions 8 times the size of their target coarse block (8,000:1,000 cells in this example). The application of local-global iteration therefore is considerably time consuming unless strong measures for adaptivity are enforced. Therefore, we put a maximum of 2-iteration limit for updating transmissibilities.

In Fig. 16 (left panel), $\delta\left(s^{\text {rec }}, s^{\text {ref }}, t\right)$ error in saturation for three algorithms of upscalingdownscaling are shown. We again observe clear improvement in the reconstructed saturation fields for ALG-MNG- compared to ALG-NG and PSM-NG. This confirms both corrections in the upscaling stage by adaptive local global method in three dimension as well as improvements in downscaling stage by modified NG. In right panel of Fig. 16, the oil flow rates are plotted and the curve for ALG-MNG- is in good agreement with that of fine reference model. However, the difference between curves of ALG-NG and ALG-MNG- is very insignificant. Therefore, the improvements in this plot are essentially due to ALG upscaling and not modified downscaling. 
Table 5 Time consumption for solution of the pressure equation by different methods for a 3D case study

\begin{tabular}{lllll}
\hline Method & $\delta P^{\mathrm{c}}$ at $1 \mathrm{PVI}$ & $\delta s^{\text {rec }}$ at $1 \mathrm{PVI}$ & $Q_{\text {oil }}$ at $1 \mathrm{PVI}\left(\mathrm{m}^{3} /\right.$ day $)$ & CPU time $(\mathrm{s})$ \\
\hline Fine & - & - & 12.17 & 331.8 \\
PSM & 0.34 & - & 17.30 & 0.037 \\
PSM-NG & 0.34 & 0.56 & 16.23 & 0.037 \\
ALG-NG & 0.12 & 0.44 & 13.35 & 54.42 \\
ALG-MNG- & 0.12 & 0.35 & 13.35 & 54.42 \\
\hline
\end{tabular}

For the analysis of computational complexity, we presented CPU time for each of methods in Table 5. The machine used is an Intel Xeon 2.67 GHz CPU processor and 4.00 GB RAM. As mentioned earlier, the CPU times are only for solution of the pressure equation so that we see a significant speed-up factor for PSM and equally for PSM-NG. In PSM, we deal with solution of very small $6 \times 6 \times 5$ systems, so that the computations drastically drop to less than a second for our machine. However, this is not the case for ALG techniques. Even with adaptivity, the calculation of corrected boundary conditions for dynamic coarse gridblocks and following iterations have undermined upscaling speed-up. Nonetheless for three dimensions, Wen et al. (2006) have shown that optimized programming in addition to modifications in the size of the extended region can lead to reasonable speed-up factors for ALG upscaling. Our emphasis however in 3D cases like previous examples still remains on the reduction of $\mathrm{NG}$ error by $\mathrm{MNG}$ and application of $\mathrm{MNG}-$.

\section{Summary}

We have introduced modifications in downscaling by NG that without computational overhead compared to the original scheme of NG can lead to better quality reconstructed saturation profiles. This modification stems from mimicking the behavior of the fine velocity distribution from an extended-local upscaling stage. In an attempt to exploit the incompressibility of waterflooding, to avoid linear solvers in NG, we showed that it is practical to only initially calculate and then reuse a set of basis functions for velocity reconstruction. The application is proved in 2D and 3D test cases as well as different sets of boundary conditions including corner-to-corner flow and directional flow with constant pressure production. We showed that use of these functions did not particularly undermine the strength of the ALG-NG upscalingdownscaling for strongly channelized systems. For the 3D case, our non-optimized code for ALG upscaling affected considerably the speed-up factor but the result for the downscaling stage in lowering the error for fine saturation profiles and the application of basis functions remained as promising as in $2 \mathrm{D}$ cases.

The study can be simply extended to other EOR processes to determine the sensitivity of downscaling or static downscaling presented here to certain physical aspects of each process. Moreover, it is very interesting to exploit advantage of the solution of the saturation equation in the same adaptive technique that velocity was reconstructed. That is to say, for each coarse gridblock, saturation is solved separate from the rest of the domain and then globally put the saturation distributions next to each other. The performance of an upscaling-downscaling can also be compared to unstructured gridding where downscaling is either replaced by grid adaptation or its quality is enhanced by an adaptive grid. In doing the latter, it is enough to perform an iteration loop based on lowering the reconstructed velocity errors to generate an optimal grid. 
Acknowledgments M. B. gratefully appreciates ESPRC-Shell Dorothy Hodgkin Postgraduate scholarship that has enabled him to undertake research at Imperial College London. Authors also thank anonymous reviewers for their invaluable comments which helped to enhance the quality of the article.

\section{References}

Aarnes, J.E.: On the use of a mixed multiscale finite element method for greater flexibility and increased speed or improved accuracy in reservoir simulation. Multiscale Model. Simul. 2, 421-439 (2004)

Aarnes, J.E., Efendiev, Y.: An adaptive multiscale method for simulation of fluid flow in heterogeneous porous media. Multiscale Model. Simul. 5(3), 918 (2006)

Arbogast, T.: Implementation of a locally conservative numerical subgrid upscaling scheme for two-phase darcy flow. Comput. Geosci. 6(3), 453-481 (2002)

Audigane, P., Blunt, M.J.: Dual mesh method for upscaling in waterflood simulation. Transp. Porous Media 55(1), 71-89 (2004)

Barker, J., Thibeau, S.: A critical review of the use of pseudorelative permeabilities for upscaling. SPE Reserv. Eng. 12(2), 138-143 (1997)

Batycky, R.P., Blunt, M.J., Thiele, M.R.: A 3D field-scale streamline-based reservoir simulator. SPE Reserv. Eng. 12(4), 246-254 (1997)

Begg, S., Carter, R., Dranfield, P.: Assigning effective values to simulator gridblock parameters for heterogeneous reservoirs. SPE Reserv. Eng. 4(4), 455-463 (1989)

Chen, Z., Hou, T.Y.: A mixed multiscale finite element method for elliptic problems with oscillating coefficients. Math. Comput. 72(242), 541-576 (2003)

Chen, Y., Durlofsky, L.J.: Adaptive local-global upscaling for general flow scenarios in heterogeneous formations. Transp. Porous Media 62(2), 157-185 (2006)

Chen, Y., Durlofsky, L.J., Gerritsen, M., Wen, X.-H.: A coupled local-global upscaling approach for simulating flow in highly heterogeneous formations. Adv. Water Resour. 26(10), 1041-1060 (2003)

Chen, Y., Durlofsky, L.J., Wen, X.-H.: Robust coarse scale modeling of flow and transport in heterogeneous reservoirs. 9th European conference on the mathematics of oil recovery, Cannes, France, vol. 30, 2004

Christie, M.: Upscaling for reservoir simulation. J. Pet. Technol. 48(11), 1004-1010 (1996)

Christie, M. A.: Flow in porous media—scale up of multiphase flow. Curr. Opin. Colloid Interface Sci. 6(3), 236-241 (2001)

Christie, M., Blunt, M.J.: Tenth SPE comparative solution project: a comparison of upscaling techniques. SPE Reserv. Eval. Eng. 4(4), 308-317 (2001)

Durlofsky, L.J.: Numerical calculation of equivalent grid block permeability tensors for heterogeneous porous media. Water Resour. Res. 27(5), 699-708 (1991)

Durlofsky, L.J.: Upscaling and gridding of fine scale geological models for flow simulation. In: 8th International Forum on Reservoir Simulation, Iles Borromees, Stresa, Italy, 2005

Efendiev, Y., Durlofsky, L.J.: A generalized convection-diffusion model for subgrid transport in porous media. Multiscale Model. Simul. 1, 504-526 (2003)

Farmer, C.L.: Upscaling: a review. Int. J. Numer. Methods Fluids 40(1-2), 63-78 (2002)

Gautier, Y., Blunt, M.J., Christie, M.A.: Nested gridding and streamline-based simulation for fast reservoir performance prediction. Comput. Geosci. 3(3), 295-320 (1999)

Gómez-Hernández, J.J., Journel, A.G.: Stochastic characterization of grid-block permeabilities: from point values to block tensors. In: Proceedings of 2nd European Conference on the Mathematics of Oil Recovery, Arles, France, pp. 83-90, 1990

Guérillot, D., Verdière, S.: Different pressure grids for reservoir simulation in heterogeneous reservoirs. Paper SPE 29148 presented at the SPE Symposium on Reservoir Simulation, San Antonio, TX 12-15 February (1995)

Holden, L., Nielsen, B.F.: Global upscaling of permeability in heterogeneous reservoirs; the output least squares (OLS) method. Transp. Porous Media 40, 115-143 (2000)

Hou, T.Y., Wu, X.-H.: A multiscale finite element method for elliptic problems in composite materials and porous media. J. Comput. Phys. 134(1), 169-189 (1997)

Jenny, P., Lee, S.H., Tchelepi, H.A.: Multi-scale finite-volume method for elliptic problems in subsurface flow simulation. J. Comput. Phys. 187(1), 47-67 (2003)

Jenny, P., Lee, S.H., Tchelepi, H.A.: Adaptive multiscale finite-volume method for multiphase flow and transport in porous media. Multiscale Model. Simul. 3(1), 50-64 (2005)

Khoozan, D., Firoozabadi, B., Rashtchian, D., Ashjari, M.: Analytical dual mesh method for two-phase flow through highly heterogeneous porous media. J. Hydrol. 400(1-2), 195-205 (2011) 
Kippe, V., Aarnes, J.E., Lie, K.A.: A comparison of multiscale methods for elliptic problems in porous media flow. Comput. Geosci. 12(3), 377-398 (2008)

Kyte, J.R., Berry, D.W.: New pseudo functions to control numerical dispersion. Soc. Pet. Eng. J. 15(4), 269276 (1975)

Lee, S.H., Wolfsteiner, C., Tchelepi, H.A.: Multiscale finite-volume formulation for multiphase flow in porous media: black oil formulation of compressible, three-phase flow with gravity. Comput. Geosci. 12(3), 351366 (2008)

Pickup, G.E., Ringrose, P.S., Jensen, J.L., Sorbie, K.S.: Permeability tensors for sedimentary structures. Math. Geol. 26(2), 227-250 (1994)

Ramé, M., Killough, J.: A new approach to flow simulation in highly heterogeneous porous media. SPE Form. Eval. 7(3), 247-254 (1992)

Renard, P., de Marsily, G.: Calculating equivalent permeability: a review. Adv. Water Resour. 20(5), 253278 (1997)

Stone, H.: Rigorous black oil pseudo functions. Paper SPE 21207 presented at the SPE Symposium on Reservoir Simulation, Anaheim, California, 17-20 February (1991)

Wen, X.-H., Gómez-Hernández, J.J.: Upscaling hydraulic conductivities in heterogeneous media: an overview. J. Hydrol. 183(1-2), ix-xxxii (1996)

Wen, X.-H., Durlofsky, L.J., Chen, Y.: Efficient 3D implementation of local-global upscaling for reservoir simulation. SPE J. 11(4), 443-453 (2006)

White, C., Horne, R.: Computing absolute transmissibility in the presence of fine-scale heterogeneity. Paper SPE 16011 presented at the SPE Symposium on Reservoir Simulation, San Antonio, Texas, 1-4 February (1987) 